\title{
Cost-Effectiveness Analysis in Comparing Alginate Silver Dressing with Silver Zinc Sulfadiazine Cream in the Treatment of Pressure Ulcers
}

\author{
Apirag Chuangsuwanich, Peerasak Chortrakarnkij, Jupaporn Kangwanpoom \\ Division of Plastic Surgery, Department of Surgery, Faculty of Medicine, Siriraj Hospital, Mahidol University, Bangkok, Thailand
}

Background The treatment of pressure ulcers is complicated, given the various wound dressing products available. The cost of different treatments varies and the cost-effectiveness of each product has not been thoroughly evaluated. We compare two wound dressing protocolsalginate silver dressing (AISD) and silver zinc sulfadiazine cream (AgZnSD) with regard to wound healing and cost-effectiveness

Methods Patients with grade III or IV sacral or trochanteric pressure ulcers were eligible for this prospective, randomized controlled trial. The patients were randomized to receive one of the two dressings for an eight-week period. The criteria of efficacy were based on the Pressure Ulcer Scale for Healing (PUSH) scoring tool. The cost of treatment was also assessed. Results Twenty patients (12 women and 8 men) were randomly assigned to receive either AISD $(n=10)$ or AgZnSD cream $(n=10)$. The demographic data and wound characteristics were comparable in the two groups. The two groups showed no significant difference in the reduction of PUSH score, wound size, or volume of exudate. The tissue type score was significantly lower in the AISD group ( $3.15 \pm 0.68-1.85 \pm 0.68$ vs. $2.73 \pm 0.79-2.2 \pm 0.41 ; P=0.015)$. The cost of treatment was significantly lower in the AISD group (377.17 vs. 467.74 USD, respectively; $\mathrm{P}<0.0001)$.

Conclusions Alginate silver dressing could be effectively used in the treatment of grade III and IV pressure ulcers. It can improve wound tissue characteristics and is cost-effective.

Keywords Pressure ulcer / Calcium alginate / Cost effectiveness
Correspondence:

Peerasak Chortrakarnkij

Division of Plastic Surgery,

Department of Surgery, Faculty of

Medicine, Siriraj Hospital,

2 Prannok road, Siriraj, Bangkok-noi,

Bangkok, Thailand 10700

Tel: $+66-24198002$

Fax: $+66-24128109$

E-mail: peerasak.si.plastic@gmail. com

This article was presented as a poster at the European Wound Management Association (EWMA) on May 25-27, 2011 in Brussels, Belgium.

The Askina Calgitrol Ag product used in this study was donated by B Braun, $\mathrm{Co}$., Ltd. of Thailand.

No potential conflict of interest relevant to this article was reported.

\section{INTRODUCTION}

Pressure ulcers are a severe type of wound phenomenon that may heavily damage the skin and surrounding tissues. The European Pressure Ulcer Advisory Panel (EPUAP) estimated the prevalence of this condition to be around $15 \%$ to $20 \%[1,2]$ of hospitalized patients in Europe. The treatment of pressure ulcer is expensive and has a considerable impact on the healthcare budget $[3,4]$. Pressure ulcers, associated with high rates of morbidity and mortality, also significantly reduce patients' quality of life while burdening healthcare workers, who must balance "best care practices" against time-constraints during their daily work.

Treatment strategies for pressure ulcers can be complicated, with a large number of different modalities and wound dressing products available $[5,6]$. Even though clear evidence exists in favor of advanced wound dressings for pressure ulcer manage- 
ment, their cost-benefit ratio remains under debate.

Among the variety of antimicrobial agents, silver has long been recognized as having the advantage of a broad antimicrobial spectrum of activity against Gram-negative and Gram-positive bacteria [7-10] with minimal development of bacterial resistance $[11,12]$. The incorporation of silver into topical dressings or as a coating on medical devices may therefore play an important role in the era of growing antibiotic resistance.

In Siriraj Hospital, silver zinc sulfadiazine cream (AgZnSD), prepared by the Siriraj Pharmacological Department, has been used as a topical dressing for open wounds such as pressure ulcers for more than 20 years with good results. Its cost is about 16.13 $\mathrm{USD} / 500 \mathrm{~g}$. The wound dressings using AgZnSD are changed once a day. The cream usually develops a film-like layer over the wound bed, which might be misinterpreted as pus or exudate.

Askina Calgitrol Ag (B Braun Hospicare Ltd., Collooney, Co. Sligo, Ireland), alginate silver wound dressing (AlSD), is a new advanced wound dressing. It is composed of a two-layer system of polyurethane foam that absorbs wound exudate and a contact layer with an alginate matrix containing silver ions that are released in a moist environment [13]. This product is a technologically advanced wound dressing that effectively combines the barrier qualities of ionic silver with the absorbency capabilities of calcium alginate and polyurethane foam. Because of its absorptive and antimicrobial properties, it is recommended for the treatment of pressure ulcer grade I-IV with proven benefits $[13,14]$.

Askina Calgitrol Ag costs about 12.9 USD/100 $\mathrm{cm}^{2}$. Since it appears to be more expensive than $\mathrm{AgZnSD}$, its use has not been accepted as a replacement for conventional topical cream. Given its cost and the necessity of assessment of the wound bed by an experienced healthcare professional, this dressing product may seem more costly as compared to the conventional dressing method with once a day dressing changes. Regarding its absorptive properties, Askina Calgitrol Ag can be left on the wound for treatment of partial thickness burns up to 3-5 days [15], which increases compliance, reduces the workload for healthcare workers, contributes to faster healing of the wound, and reduces the cost burden to patients who have to pay for the treatment of pressure ulcers.

Thus, a proper cost-effectiveness analysis of this product could have some impact on the improvement of treatment policy, while helping to reduce the treatment costs for chronic diseases associated with wounds. The purpose of this study was to analyze the cost-effectiveness of alginate silver dressing compared with silver zinc sulfadiazine cream in the treatment of pressure ulcers.

\section{METHODS}

\section{Patient population}

A prospective controlled trial, following the CONSORT statement of 2010 [16], was conducted between October 2009 and December 2010 in the outpatient unit, of the Department of Surgery, Siriraj Hospital, Mahidol University, Thailand. The present study was approved by the Siriraj Institutional Review Board.

The inclusion criteria consists of the following: 1) Patients suffering from pressure ulcer(s) in the sacral or trochanteric area. 2) Those with pressure ulcers of grades ranging from III to IV [17] according to the NPUAP pressure ulcer staging system. 3) Cooperation of the patient or her/his relatives who could complete the consent form and have regular weekly visits according to the study protocol. 4) Age $>20$ years old.

The exclusion criteria consists of the following: 1) Pressure ulcers with necrotic tissue that could not be managed with adequate debridement. 2) Pressure ulcers with clinical evidence of apparent infection, e.g.; ulcers surrounded by an advancing, indurated red border, warm or tender, with purulent exudate, or accompanied by a bad odor $[18,19]$. 3) Patients with a known history of hypersensitivity to any part of the drugs or products used in this study including calcium alginate, polyurethane foam, or silver derivatives. 4) Patients with a known history of hypersensitivity to sulfa derivatives. 5) Patients with a history of glucose-6-phosphate dehydrogenase deficiency [20].

\section{Sample size estimation}

The number of patients required in this study was estimated by using the following equation:

$$
n=\left[\left(Z_{\alpha / 2} \cdot S D\right) / d\right]^{2}
$$

where n, population number per group; SD, standard deviation; $d$, margin of error; $\alpha$, probability of type I error.

The primary outcome of this study was healing of the ulcer, and the Pressure Ulcer Scale for Healing (PUSH) score was the indicator of the healing. The standard deviation of the PUSH score from the previous similar study was about 3 . The margin of error meant the least difference in the PUSH score that could be detected clinically, which was about 2 . The probability of a type I error, which was two-sided, equaled 0.05. After completion of the equation, $\mathrm{n}$ was equal to 8.64 , which meant that this study required at least 9 patients per group for the protocol to produce clinically significant results. We decided to collect 13 patients per group because of the high chance of loss to follow- 
up during the study period.

\section{Study design and wound dressing protocol}

The enrolled patients were randomly divided into two groups by drawing from a sealed envelope for each group. The population consisted of an AlSD-treated group and an AgZnSD-treated group. The study period was eight weeks for each patient. The data were compared with regard to the patients' demographic data. In the AlSD-treated group, the treatment consisted of the application of the AlSD, which was changed every 3 days until completion of the study. The AgZnSD group treatment consisted of the application of silver zinc sulfadiazine cream. The dressing was changed once a day and dry gauze was placed as an outer dressing. Each patient was scheduled for a weekly visit until completion of the eight-week period for the study. The wounds were debrided as necessary to remove all necrotic tissue.

\section{Evaluation and assessment criteria}

Demographic data including age, gender, grade and location of the wound, number of wound dressing changes per eight-week period, number of debridement procedures, and any co-morbid condition were collected. At the beginning of the present study, the wound size was determined using the VISITRAK wound measurement system, and wound photography, and a bacterial wound culture was collected. The pressure ulcer characteristics and healing rate were assessed using the PUSH ver. 3.0 score [21]. The PUSH score ranged from 0 to 17 based on the ulcer surface area (length $\times$ width), exudate amount, and tissue type characteristics. A reduction in the PUSH score was regarded to be an indicator of ulcer healing. After the wound was cleansed by nurse, it was examined and scored by an independent operator, plastic surgeon, who was blinded to the dressing protocol. The wound was measured and evaluated for changes in the wound size, grade, tissue characteristics, and amount of exudate. The photography of the wound bed was recorded. Then, the wound dressing was performed according to the protocol for each group of patients.

The cost of treatment was recorded. The average wound dressing unit cost (DUC) was about 8.06 USD, plus 16.13 USD for each debridement procedure. The cost of the AgZnSD, which could be reimbursed by the government, was included in the DUC according to hospital policy. The cost of the AlSD product was calculated in the case of using the smallest available size of the product, $10 \times 10 \mathrm{~cm}^{2}, 15 \times 15 \mathrm{~cm}^{2}$, and $20 \times 20 \mathrm{~cm}^{2}$, that could be used to adequately cover the wound bed. This product costs about 12.90, 25.80, and 48.39 USD for $10 \times 10 \mathrm{~cm}^{2}$, $15 \times 15 \mathrm{~cm}^{2}$, and $20 \times 20 \mathrm{~cm}^{2}$, respectively.

\section{Statistical analysis}

The data was analyzed using SPSS ver. 16.0 (SPSS, Chicago, IL, USA). The patients' characteristics, clinical data, and cost factor expressed in physical units were compared using the chisquared $\left(\chi^{2}\right)$ test for categorical data and the Student's t- test for normally distributed quantitative variables. As they had not been normally distributed, the data were compared using the Mann-Whitney nonparametric test. The P-values less than 0.05 were considered significant.

\section{Uncertainty of the cost-effectiveness ratio \\ Sensitivity analysis}

The dressing unit cost, which was the main part of the total cost of treatment in each group, varies among hospitals. In Siriraj Hospital, the average DUC was about 8.06 USD. Alteration in the DUC may affect the confidence of cost-effectiveness result of this study.

A one-way sensitivity analysis was performed on one parameter used in analysis, the "dressing unit cost". We hypothesized that alterations in the DUC would not affect the cost-effectiveness result. In the univariate sensitivity analyses, the parameter of DUC was increased or decreased by multiples of 10 percent to determine the main cost and effect drivers in our model. The result of changing the DUC was shown in the incremental and decremental cost-effectiveness analysis model.

\section{RESULTS}

Between October 2009 and December 2010, a total of 26 patients met the above criteria and were included in this study. Six patients were excluded because of death during the study $(n=2)$, taking oral anticoagulants so that the necrotic tissue of the wound could not be debrided adequately $(n=3)$, and an active infection at the peri-wound area $(n=1)$. In the end, 20 patients were enrolled in the study (Fig. 1).

All of the 20 patients were randomly divided into the two groups according to the study protocol. Ten patients in each group including 15 wounds in the AlSD group and 13 wounds in the AgZnSD group completed the eight-week period of the study. The mean age was $76 \pm 7.88$ and $73.3 \pm 7.27$ years in the AlSD and AgZnSD groups, respectively. The demographic data of the patients in each group, including the age, sex, body mass index (BMI), grade and location of the wound, and associated co-morbidities such as mellitus, hypertension, dyslipidemia, and old cerebrovascular accident were compared (Table 1). From the univariate analysis, no statistically significant differences between the two groups could be identified.

The mean ulcer area at the beginning of treatment was 


\section{Fig. 1. Flow diagram of the study population}

The flow diagram shows the allocation pattern of the population. Twenty-six patients were eligible in this study; four were excluded due to anticoagulant medication $(n=3)$ and, one was excluded because of an active infection around the pressure ulcer $(n=1)$. Two patients died during the study.

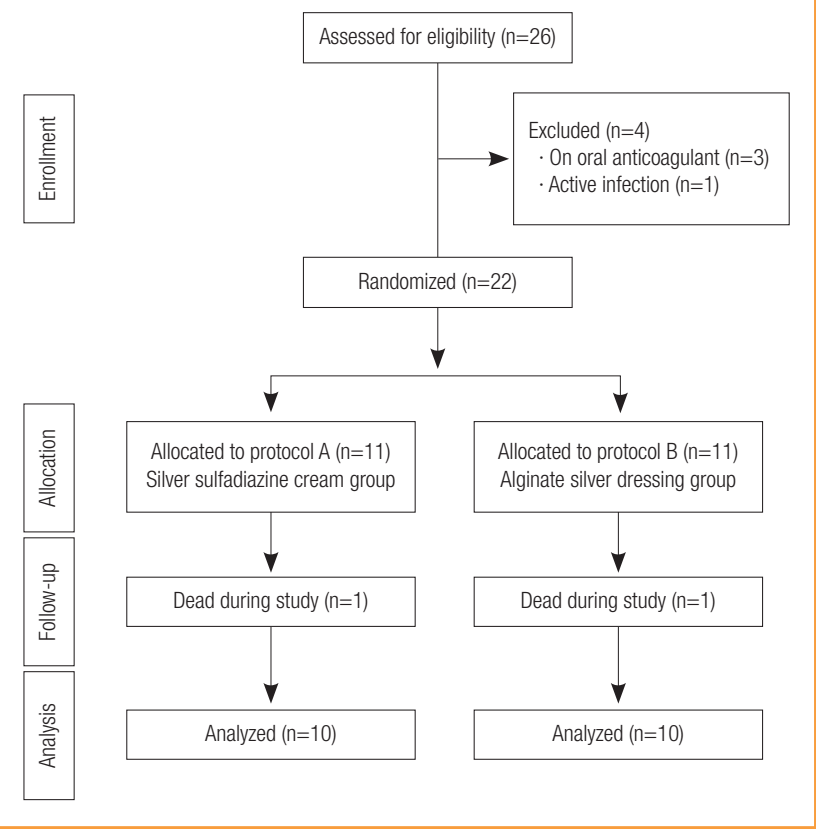

Table 1. Baseline characteristics of patients, and grade and location of the wound in AISD and AgZnSD groups

\begin{tabular}{|c|c|c|c|}
\hline Characteristic & $\begin{array}{l}\text { AISD-treated } \\
\text { group }(n=10)\end{array}$ & $\begin{array}{c}\text { AgZnSD-treated } \\
\text { group }(n=10)\end{array}$ & P-value \\
\hline Age & $76 \pm 7.88$ & $73.3 \pm 7.27$ & 0.437 \\
\hline Sex (male:female) & $4: 6$ & $4: 6$ & 1.000 \\
\hline $\mathrm{BMl}$ & $21.61 \pm 2.03$ & $23.58 \pm 2.4$ & 0.064 \\
\hline Diabetes & 5 & 2 & 0.350 \\
\hline Hypertension & 2 & 4 & 0.628 \\
\hline Dyslipidemia & 1 & 3 & 0.582 \\
\hline Old CVA & 6 & 5 & 1.000 \\
\hline Pressure ulcer grade & & & 0.718 \\
\hline III & 8 & 8 & \\
\hline IV & 5 & 7 & \\
\hline Pressure ulcer location & & & 1.000 \\
\hline Sacrum & 9 & 10 & \\
\hline Right trochanteric & 2 & 4 & \\
\hline Left trochanteric & 2 & 1 & \\
\hline \multicolumn{4}{|c|}{$\begin{array}{l}\text { Values are presented as mean } \pm \text { standard deviation. } \\
\text { AISD, alginate silver dressing; AgZnSD, silver zinc sulfadiazine cream; BMl, body } \\
\text { mass index; CVA, cerebrovascular accident. }\end{array}$} \\
\hline
\end{tabular}

$36.11 \mathrm{~cm}^{2}$ and $35.50 \mathrm{~cm}^{2}$ in the AlSD and AgZnSD groups, respectively $(\mathrm{P}=0.961)$. At the end of treatment, the wound area of the experimental and control group were reduced by $44.27 \%$ vs. $51.07 \%$, respectively ( $p=0.504$ ) (Fig. 2 ). The mean PUSH score at the beginning of treatment was $14 \pm 2.3$ and

\section{Fig. 2. Wound area}

This representative figure shows the cumulative improvement in the wound area $\left(\mathrm{cm}^{2}\right)$ by subject over time (weeks). A wound size reduction was found in both groups with no statistically significant difference $(P=0.504)$. AISD, alginate silver dressing; $A g Z n S D$, silver zinc sulfadiazine cream.

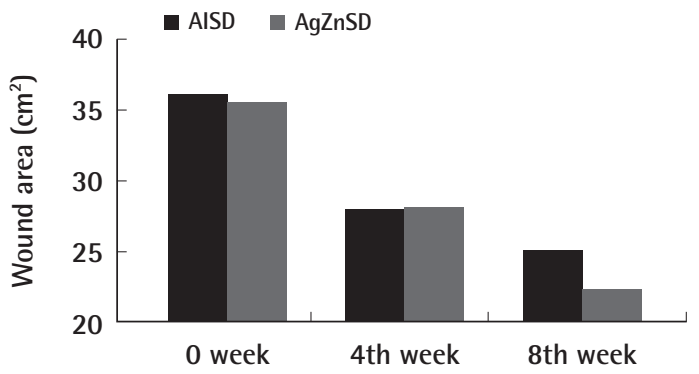

\section{Fig. 3. PUSH scores}

This representative figure shows the cumulative improvement in the PUSH score by subject over time (weeks). A PUSH score reduction was found in both groups with no statistically significant difference $(P=0.402)$. PUSH, Pressure Ulcer Scale for Healing; AISD, alginate silver dressing; AgZnSD, silver zinc sulfadiazine cream.

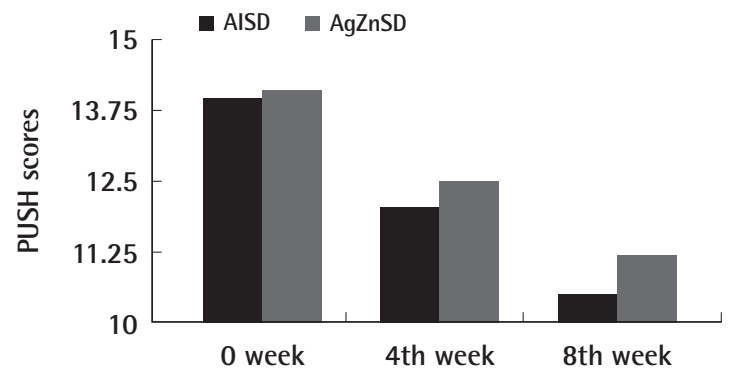

$14.13 \pm 1.59$ in the AlSD and AgZnSD groups, respectively. The PUSH score was reduced to $10.5 \pm 3.7$ vs. $11.20 \pm 2.9$, respectively $(\mathrm{P}=0.402)$ (Fig. 3).

For the exudate amount, the initial score of the exudate amount was $2.07 \pm 0.86$ vs. $1.86 \pm 0.92$ in the AlSD and AgZnSD group, respectively $(\mathrm{P}=0.539)$. At the end of the eighth week, the score of the exudate amount was reduced to $1.38 \pm 0.65$ vs. $1.2 \pm 0.94$ in the AlSD and AgZnSD group, respectively ( $\mathrm{P}=0.557)$ (Fig. 4). However, in an analysis of tissue type, the initial score for the tissue type was $3.15 \pm 0.68$ vs. $2.73 \pm 0.79$ in the AlSD and Ag$\mathrm{ZnSD}$ group, respectively. At the end of the study, the score was reduced to $1.85 \pm 0.68$ vs. $2.2 \pm 0.41$, respectively. The reduction in the tissue type score was statistically significantly better in the AlSD group than the AgZnSD group $(\mathrm{P}=0.015)$ (Fig. 5). The bacteriological studies of the AISD group are shown in Table 2.

The cost of treatment was calculated according to the following factors: 1) Dressing unit cost: including the dressing change workload, gauze, and AgZnSD cream. This part cost ap- 


\section{Fig. 4. Exudate amount}

This representative figure shows the cumulative improvement in exudates amount by subject over time (weeks). The exudate amount was reduced in both groups with no statistically significant difference $(P=0.557)$. AISD, alginate silver dressing; AgZnSD, silver zinc sulfadiazine cream.

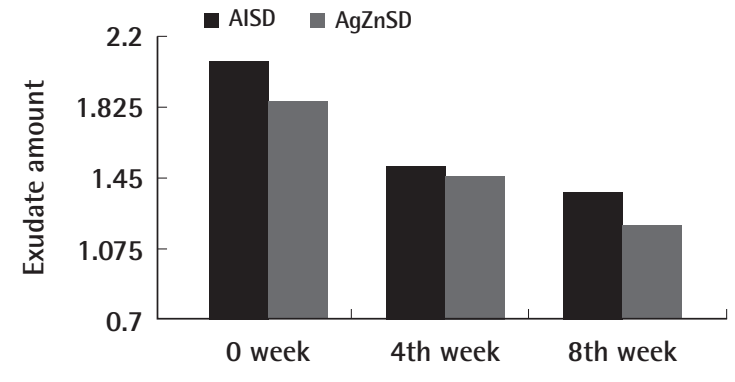

\section{Fig. 5. Tissue type}

This representative figure shows the cumulative improvement in the tissue type by subject over time (weeks). The tissue type was improved in both groups with a statistically significant improvement in the alginate silver wound dressing (AISD) group $(P=0.015)$. AgZnSD, silver zinc sulfadiazine cream.

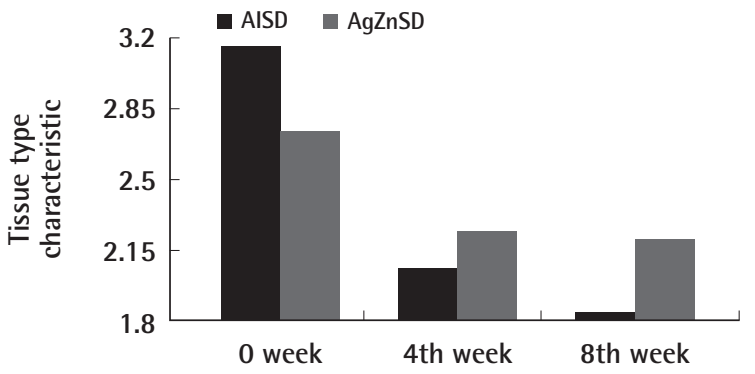

Table 2. Microbiological study (alginate silver dressing group)

\begin{tabular}{|c|c|c|}
\hline Patient & Week 0 & Week 8 \\
\hline A & $\begin{array}{l}\text { Few polymorphonuclear cells, } \\
\text { no bacteria seen }\end{array}$ & No growth \\
\hline \multirow[t]{2}{*}{ B } & Pseudomonas aeruginosa-few & Moderate commensal flora \\
\hline & Providencia struartii-few & \\
\hline C & Mixed bacteria-numerous & Moderate commensal flora \\
\hline D & Mixed bacteria-numerous & No growth \\
\hline $\mathrm{E}$ & $\begin{array}{l}\text { Staphylococcus species. } \\
\text { (coagulase negative )-few }\end{array}$ & Few commensal floral \\
\hline $\mathrm{F}$ & $\begin{array}{l}\text { Enterobacter cloacae-moderate } \\
\text { Acinetobacter baumannii-moderate }\end{array}$ & No growth \\
\hline G & No growth & No growth \\
\hline H & No growth & \\
\hline । & Escherichia coli, ESBL negative-few & No growth \\
\hline J & $\begin{array}{l}\text { Escherichia coli, ESBL negative-few } \\
\text { Proteus mirabilis, ESBL negative-few }\end{array}$ & Proteus mirabilis-few \\
\hline
\end{tabular}

\section{Fig. 6. Cost of treatment}

This representative figure shows the comparison of cost of treatment between the two groups. At the end of the study, the cost of treatment in the alginate silver wound dressing (AISD) group was reduced compared to the silver zinc sulfadiazine cream (AgZnSD) group with a statistically significant difference $(\mathrm{P}<0.001)$.

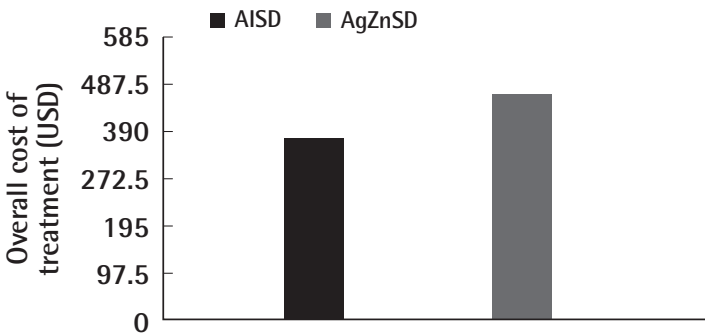

\section{Fig. 7. Cost of treatment and dressing unit cost}

This figure shows the relationship between the dressing unit cost and overall cost of treatment. Increments and decrements in multiples of $10 \%$ of the dressing unit cost affected the total cost of treatment. The total cost of treatment of the silver zinc sulfadiazine cream (AgZnSD) group was greater than that of the alginate silver wound dressing (AISD) group unless the dressing unit cost was reduced by more than $20 \%$.

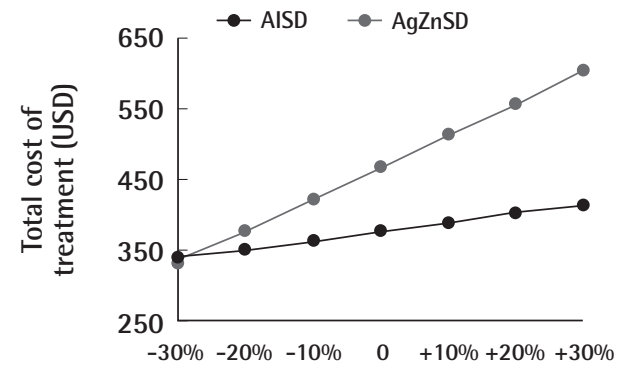

proximately 8.06 USD/dressing change. 2) Debridement cost including the workload, surgical instruments, and procedural charge. This cost about 16.13 USD/procedure. 3) Alginate silver dressing product cost: about $12.9 \mathrm{USD} / 10 \times 10 \mathrm{~cm}^{2}, 25.8$ USD $/ 15 \times 15 \mathrm{~cm}^{2}$, and $48.39 \mathrm{USD} / 20 \times 20 \mathrm{~cm}^{2}$ dressing.

After the treatment period of 8 weeks, the average overall cost of treatment was 377.17 vs. 467.74 USD in the AlSD and AgZnSD groups, respectively $(\mathrm{P}<0.001)$ (Fig. 6).

The result of a sensitivity analysis performed for the dressing unit cost incremented and decremented from 8.06 by multiples of $10 \%$ are shown in (Fig. 7). The cost of treatment between the two groups was statistically significant above a $-10 \%$ value of the DUC $(-10 \%, \mathrm{P}=0.027 ;+10 \%, \mathrm{P}<0.001 ;+20 \%, \mathrm{P}<0.001$; and $+30 \%, \mathrm{P}<0.001)$. Statistical significance was absent at values $-20 \%(\mathrm{P}=0.306)$ and at $-30 \%(\mathrm{P}=0.775)$. No complications from the treatment were recorded for either group. 


\section{Fig. 8. Case study 1}

A 84-year-old female with a grade III sacral pressure ulcer. She had underlying hypertension, dyslipidemia, and old ischemic stroke with a totally dependent status. She had suffered from a sacral pressure ulcer for 5 months. She was treated with alginate silver dressing. After an eight-week period of treatment, the wound size had been reduced from 20 to $12 \mathrm{~cm}^{2}$. (A) At the first week. (B) At the second week. (C) At the fourth week. (D) At the eighth week.
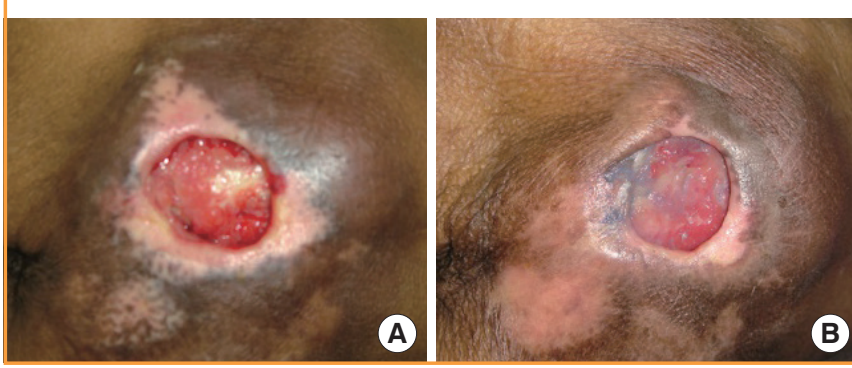

\section{Fig. 9. Case study 2}

An 80-year-old female with a grade III sacral pressure ulcer. She had underlying of diabetes mellitus. She suffered from a sacral pressure ulcer with necrotic tissue for 15 days. She received debridement, and the wound was treated with alginate silver dressing. Eight weeks after treatment, the wound size was reduced from 20 to $3 \mathrm{~cm}^{2}$. (A) At the first week before debridement with some necrotic tissue. (B) At the eighth week.
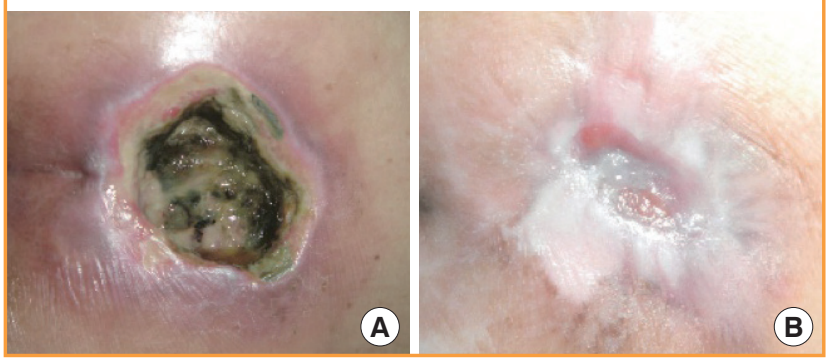

\section{Case 1}

An 84-year-old female with underlying hypertension, dyslipidemia, and old ischemic stroke (Fig. 8). She had suffered from a grade III sacral pressure ulcer for 5 months. She was treated with alginate silver dressing. Eight weeks after treatment, the wound size had been reduced from $20 \mathrm{~cm}^{2}$ to $12 \mathrm{~cm}^{2}$.

\section{Case 2}

An 80-year-old female with underlying diabetes mellitus (Fig. 9). She had had a grade III sacral pressure ulcer with some necrotic tissue for 15 days. She received debridement and the wound was treated with alginate silver dressing. Eight weeks after treatment, the wound size had been reduced from $20 \mathrm{~cm}^{2}$ to $3 \mathrm{~cm}^{2}$.

\section{DISCUSSION}

Pressure ulcers remain a common problem among in-patient and out-patient conditions throughout the world. They usually occur in seriously ill, debilitated, and dependent patients. The prevalence rate ranges from $3 \%$ to $66 \%$ in hospitals, nursing
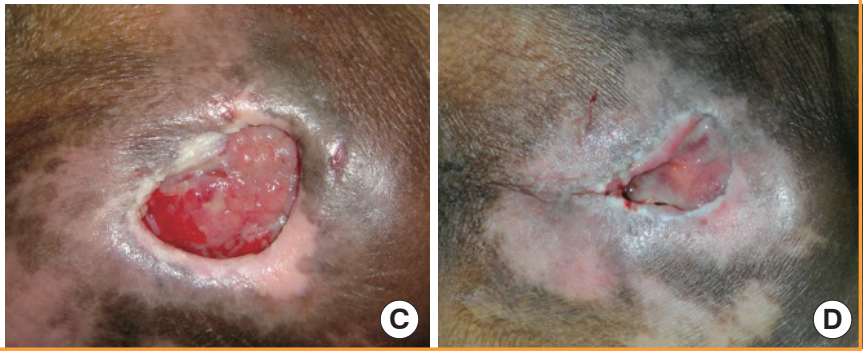

homes, and home care $[1,2]$. Silver has been used in wound care, given its long history of use and broad antimicrobial spectrum properties. The conventional method of dressing for stage III and IV pressure ulcers in our hospital is cleansing the wound with normal saline solution and applying silver zinc sulfadiazine cream over the wound surface once a day. This may present a significant burden to the patients and caregivers for the timeconsuming treatments, and the necessity for patient's transportation to reach medical personnel.

Many new dressing products containing silver have entered the market. One product in a group of alginate silver dressings (Askina Calgitrol Ag, B Braun Hospicare Ltd., Collooney, Co.) is a two-layer wound dressing composed of a polyurethane foam that absorbs wound exudate and a contact layer with an alginate matrix containing silver ions that are released in a moist environment [13]. Regarding its antimicrobial properties, an in vitro study demonstrated sustained and consistent silver ion release in a simulated wound fluid setting over a specified time period, and it has been shown to be effective against a wide range of human clinical isolates and also against resistant organisms like methicillin-resistant staphylococcus aureus (MRSA) [1012]. Because of its softness, pliability, and absorptive properties, this type of dressing is well suited for the treatment of pressure ulcers, which usually present in shallow to deep cavities with moderate to heavy exudate. Although alginate silver dressing is recommended for wounds that may show critical colonization or evidence of infection, the cost-benefit tradeoff of its uses are still under debated.

Following the results of this study, alginate silver dressing can be used for the treatment of pressure ulcers with a better wound healing profile than conventional treatment, but without a significant difference in wound size reduction $(\mathrm{P}=0.504)$ or $\mathrm{PUSH}$ score $(\mathrm{P}=0.402)$. Furthermore, if only changes in the tissue type characteristics are considered, the study group experienced significantly greater improvement (reduction of scores) than 
the control group. Specifically, the mean tissue type score was reduced from $3.15 \pm 0.68$ to $1.85 \pm 0.68$ in the AlSD group and from $2.73 \pm 0.79$ to $2.2 \pm 0.41$ in the $A g Z n S D$ group $(P=0.015)$.

This study showed that alginate silver dressing is a cost-minimizing treatment modality. It can also reduce the numbers of dressing changes along the course of treatment. It may help to improve the quality of life because patients or caregivers could spend less time caring for wounds or travelling to meet a care provider for dressing changes. The overall costs of treatment were also reduced (377.17 USD for AlSD vs. 467.74 USD for AgZnSD, respectively) in favor of the AlSD group $(\mathrm{P}<0.001)$.

According to the total cost of treatment, the main factor is the dressing unit cost, which contributed to about $80 \%$ of the overall cost of treatment in the AlSD group and $>90 \%$ in the AgZnSD group. Changes in the price of the dressing unit cost will directly affect the overall cost of treatment. To enhance the confidence of this study, we hypothesized that alterations in the DUC would not affect the cost-effectiveness of the treatment modality. The sensitivity analysis confirmed our hypothesis for a reduction of the DUC by not more than $20 \%$. In such cases, the alginate silver dressing could still have a significant difference in cost reduction in the AlSD group. When we reduced the DUC by more than $20 \%$, the difference in the overall cost of treatment was not statistically significant. This means that if the DUC was reduced to below 6.45 USD, the overall cost of treatment between the AlSD and AgZnSD groups would not differ significantly.

There are many factors that influence the wound healing, including relieving the pressure, improvement of nutritional status, the patient's general condition, and wound management. Most of these factors appear difficult to be control, but could be managed with proper randomization. The main limitation of our study comes from the relatively small number of patients included in the evaluation, which may raise some concerns about the statistical power of our comparison. This study, even with the small number of subjects, showed that alginate silver dressing can provide a comparable wound healing rate at lower costs of treatment. Our study helps clarifying based on real-practice data that alginate silver dressings indeed provide cost savings in the treatment of pressure ulcers. The data suggest that similar cost-effectiveness analyses should be carried out for other clinical indications of alginate silver dressings.

Alginate silver dressings could be used in the treatment of grade III and IV sacral and trochanteric pressure ulcers. They contributed to good wound healing rates when compared to the standard treatment and even improved the tissue type characteristics. Furthermore, they could reduce the numbers of dressing changes and the overall cost of treatment.
The alginate silver dressing used in this study was Askina Calgitrol Ag (B. Braun Hospicare Ltd., Collooney, Co.).

\section{REFERENCES}

1. Vanderwee K, Clark M, Dealey C, et al. Pressure ulcer prevalence in Europe: a pilot study.J Eval Clin Pract 2007;13:22735.

2. Tannen A, Dassen T, Bours G, et al. A comparison of pressure ulcer prevalence: concerted data collection in the Netherlands and Germany. Int J Nurs Stud 2004;41:607-12.

3. Bennett G, Dealey C, Posnett J. The cost of pressure ulcers in the UK. Age Ageing 2004;33:230-5.

4. Xakellis GC, Frantz R. The cost of healing pressure ulcers across multiple health care settings. Adv Wound Care 1996; 9:18-22

5. Inman KJ, Sibbald WJ, Rutledge FS, et al. Clinical utility and cost-effectiveness of an air suspension bed in the prevention of pressure ulcers. JAMA 1993;269:1139-43.

6. Reddy M, Gill SS, Kalkar SR, et al. Treatment of pressure ulcers: a systematic review. JAMA 2008;300:2647-62.

7. Mooney EK, Lippitt C, Friedman J, et al. Silver dressings. Plast Reconstr Surg 2006;117:666-9.

8. Lansdown AB. Silver. I: Its antibacterial properties and mechanism of action. J Wound Care 2002;11:125-30.

9. Hermans MH. Silver-containing dressings and the need for evidence. Adv Skin Wound Care 2007;20:166-73.

10. Thomas S, McCubbin P. An in vitro analysis of the antimicrobial properties of 10 silver-containing dressings. J Wound Care 2003;12:305-8.

11. Hooper SJ, Williams DW, Thomas DW, et al. An in vitro comparison of two silver-containing antimicrobial wound dressings. Ostomy Wound Manage 2012;58:16-22.

12. Percival SL, Slone W, Linton S, et al. The antimicrobial efficacy of a silver alginate dressing against a broad spectrum of clinically relevant wound isolates. Int Wound J 2011;8:237-43.

13. Ricci KE, Pittarello M, Cassino R, et al. AskinaCalgitrol $\mathrm{Ag}^{\oplus}$ : clinical use of an advanced ionic silver dressing. Acta Vulnologica 2008;5:105-11.

14. Addison D, Rennison TJ, Del Bono M. The antimicrobial properties of a silver alginate dressing for moderate to heavily exuding, wounds: 688. J Wound Ostomy Continence Nurs 2005;32:S28.

15. Opasanon S, Muangman P, Namviriyachote N. Clinical effectiveness of alginate silver dressing in outpatient management of partial-thickness burns. Int Wound J 2010;7:467-71.

16. Schulz KF, Altman DG, Moher D, et al. CONSORT 2010 statement: updated guidelines for reporting parallel group 
randomised trials. BMJ 2010;340:c332.

17. Black J, Baharestani MM, Cuddigan J, et al. National Pressure Ulcer Advisory Panel's updated pressure ulcer staging system. Adv Skin Wound Care 2007;20:269-74.

18. Livesley NJ, Chow AW. Infected pressure ulcers in elderly individuals. Clin Infect Dis 2002;35:1390-6.

19. Parish LC, Witkowski JA. The infected decubitus ulcer. Int J
Dermatol 1989;28:643-7.

20. Fuller FW. The side effects of silver sulfadiazine. J Burn Care Res 2009;30:464-70.

21. Stotts NA, Rodeheaver GT, Thomas DR, et al. An instrument to measure healing in pressure ulcers: development and validation of the pressure ulcer scale for healing (PUSH). J Gerontol A Biol Sci Med Sci 2001;56:M795-9. 